\title{
Effects of Karate Fights on Achilles Tendon Stiffness Measured by Myotonometry
}

\author{
by \\ Beata Pożarowszczyk ${ }^{1}$, Weronika Pawlaczyk ${ }^{1}$, Małgorzata Smoter4, \\ Andrzej Zarzycki ${ }^{5}$, Dariusz Mroczek², Martyna Kumorek ${ }^{1}$, Kazimierz Witkowski, \\ Kawczyński Adam ${ }^{1}$
}

The aim of the study was to investigate the influence of karate fights on Achilles tendon stiffness in karate competitors. Eleven male karate fighters participated in the present study. A handheld MyotonPRO device (MyotonPRO, Myoton Ltd, Estonia) was applied to measure Achilles tendon stiffness in karate fighters. The Achilles tendon was tested $5 \mathrm{~cm}$ above the tuber calcanei. Stiffness measurements were performed before and after eight sparring fights. Each fight lasted $2 \mathrm{~min}$ and was separated by a $2 \mathrm{~min}$ rest period. Achilles tendon stiffness for the dominant leg increased significantly from before fights $(751.57 \pm 123.493 \mathrm{~N} / \mathrm{m})$ to immediately after fights $(809.43 \pm 160.425 \mathrm{~N} / \mathrm{m})$ $(p=0.012)$. Presented results should be used by strength and conditioning coaches in training programs as a way to decrease the risk of injury.

Key words: tendon stiffness, karate, myotonometry.

\section{Introduction}

Karate is a contact sport with high possibilities of injuries especially during sparring or competition. Karate training consists of three main elements: kihon, kata and kumite. Sterkowicz and Sterkowicz-Przybycień (2013) in their research showed that in karate lower limb injuries, including Achilles tendon (AT), were the most frequent. In the Kujala et al.'s (1995) study, the total number of injuries was much higher during training sessions than the rate of injuries occurring during competitions. The AT due to its function experiences significant mechanical loads by storing and returning elastic energy and enabling explosive movements such as running and jumping (Komi et al., 1992; Park et al., 2006).
Previously, Foure et al. (2012) used ultrasonography combined with dynamometry and electromyography to assess muscle-tendon (gastrocnemii muscle and AT) complex passive stiffness. In another study authors measured the AT cross-sectional area by ultra-sonographic imaging scans (Foure et al., 2013). Those methods were very precise, but could be used only in laboratory settings. That is why it seems to be reasonable to apply some alternative method in assessing tendon and muscles viscoelastic properties. Additionally, there is a lack of myotonometric studies on athletes in recent literature. We could find only few relevant studies, mostly in clinical approaches i.e. muscle

\footnotetext{
1 - University School of Physical Education, Department of Paralympics Sports, Wroctaw, Poland.

2 - University School of Physical Education, Department of Athletes Motor Skills, Wroctaw, Poland.

3 - University School of Physical Education, Department of Sport Didactics, Wroctaw, Poland.

4. University School of Physical Education, Department of Biostructure, Wroctaw, Poland.

5 - Wrockaw University of Environmental and Life Sciences, Wroclaw, Poland.
} 
belly and tendon in patients with Parkinson disease (Marusiak et al., 2011) and biceps and triceps brachii muscles in patients with subacute stroke (Chuang et al., 2012). It should be underlined that the muscle-tendon complex (gastrocnemii muscle and AT) plays an important role in each sport due to its role in the elastic energy storage-recoil process (Foure et al., 2013). Moreover, the tendon dissipation coefficient, which is representative for a storage-recoil process, was shown to be altered by plyometric training in vivo (Foure et al., 2012). The ability of the AT to use elastic energy is crucial for explosive performance in athletes (Wang et al., 2012). Complete AT ruptures are found often in athletes who participate in sports involving explosive acceleration or maximal effort (Retting et al., 2005). In that case, precise investigation of AT elastic properties seems even more necessary.

Myotonometry has become increasingly popular in assessing viscoelastic properties of muscles; moreover, it can be used before and after training or physiotherapy treatment, as it is a noninvasive technique and the device is portable and easy to operate (Ditroilo et al., 2011; Marusiak et al., 2011).

To the best of our knowledge, no study has addressed the effect of karate fights on AT stiffness, assessed by myotonometry. The establishment of specific adaptations of tendon and muscle mechanical properties as the effect of specific loads during karate fights may provide important information which could be applied in designing training loads and injury prevention programs.

Therefore, the aim of the present study was to investigate the influence of karate fights on AT stiffness occurring in karate competitors. We hypothesized that there would be an increase in AT stiffness in karate competitors in response to a series of fights.

\section{Material and Methods}

\section{Participants}

Eleven male karate fighters (aged $24.7 \pm$ 4.5 years; body height, $176.4 \pm 65.8 \mathrm{~cm}$; body mass, $72.3 \pm 5.4 \mathrm{~kg}$ ) participated in the present study. All subjects were present at the Karate Shotokan Camp and preparing for the Polish and European Championships. Informed consent was obtained from each subject. The study was approved by ethics committee of the University School of Physical Education in Wrocław and conducted in accordance with the Declaration of Helsinki.

Measures

A handheld MyotonPRO device (MyotonPRO, Myoton Ltd, Estonia) was applied to measure AT stiffness in karate fighters. The AT was tested $5 \mathrm{~cm}$ above the tuber calcanei. Additionally, the middle point of the soleus muscle belly was tested. For this study, a pattern of five measurements on each tested point on the tendon and muscle was used and the mean was calculated for further analysis. Tendon and muscle stiffness is a biomechanical property that characterizes resistance to a contraction or to an external force that deforms its initial shape. The calculation formula for tendon and muscle stiffness was as follows: $\mathrm{S}=\mathrm{amax} \cdot \mathrm{m}_{\text {probe }} / \Delta \mathrm{l} ; \mathrm{a}-$ acceleration of the damped oscillation; $\mathrm{m}_{\text {probe - }}$ mass of the measurement mechanism, $\Delta \mathrm{l}-$ maximal displacement of the tissue (MyotonPRO, Myoton Ltd, Estonia).

Procedures

Measurements were performed before and after eight kumite (sparring fights with an opponent). Each fight lasted $2 \mathrm{~min}$ and was separated by a $2 \mathrm{~min}$ rest period during which subjects lay on a settee with muscles relaxed; feet were out of the edge of the settee. The dominant leg was indicated by observation of the subjects during kumite (based on the fight position); moreover, it was confirmed by an interview with the subjects.

\section{Statistics}

Two one-way ANOVAs (repeatedmeasure analysis of variance) with one factor (time of measurements) were used to analyze tendon and muscle stiffness results. The normality of the data distribution was checked by the Shapiro-Wilk test. In all tests, $p \leq 0.05$ was considered significant, and partial $\eta^{2}$ was calculated to assess effect sizes. The data are presented as mean and standard error of mean (SEM) in the text.

\section{Results}

AT stiffness of the dominant leg increased significantly from before fights (751.6 \pm $123.5 \mathrm{~N} / \mathrm{m})$ to immediately after fights $(809.4 \pm$ $160.4 \mathrm{~N} / \mathrm{m})\left(p=0.012 ; \eta^{2}=0.4\right)$. Stiffness of soleus 
muscle of the dominant leg decreased significantly from before $(452.2 \pm 45.7 \mathrm{~N} / \mathrm{m})$ to immediately after the fights $(425.3 \pm 50.8 \mathrm{~N} / \mathrm{m})$ $\left(p=0.001 ; \eta^{2}=0.7\right)$ (Table 1$)$.

For the AT of the non-dominant leg, there were no significant changes for stiffness measurements performed before $(813.8 \pm 134.6$ $\mathrm{N} / \mathrm{m}$ ) and immediately after the fights
$(831.7 \pm 168.2 \mathrm{~N} / \mathrm{m}) \quad\left(p=0.6 ; \eta^{2}=0.02\right)$. For the soleus muscle of the non-dominant leg, there were no significant changes for stiffness measurements performed before $(438.8 \pm 164.7 \mathrm{~N} / \mathrm{m})$ and immediately after the fights $(440.3 \pm 158.1 \mathrm{~N} / \mathrm{m})(p$ $\left.=0.7 ; \eta^{2}=0.03\right)($ Table 1$)$.

\section{Table 1}

Achilles tendon and soleus muscle stiffness before and after eight 2 min karate fights.

\begin{tabular}{lcccc}
\hline Session/location & $\begin{array}{c}\text { Achilles tendon } \\
\text { stiffness }[\mathrm{N} / \mathrm{m}] \\
\text { dominant leg }\end{array}$ & $\begin{array}{c}\text { Achilles tendon } \\
\text { stiffness [N/m] } \\
\text { non-dominant leg }\end{array}$ & $\begin{array}{c}\text { Soleus muscle } \\
\text { stiffness [N/m] } \\
\text { dominant leg }\end{array}$ & $\begin{array}{c}\text { Soleus muscle } \\
\text { stiffness [N/m] } \\
\text { non-dominant leg }\end{array}$ \\
\hline before fights & $751.6 \pm 123.5$ & $813.8 \pm 134.6$ & $452.2 \pm 45.7$ & $438.8 \pm 164.7$ \\
after fights & $809.4 \pm 160.4^{*}$ & $831.7 \pm 168.2$ & $425.3 \pm 50.8^{*}$ & $440.3 \pm 158.1$ \\
\hline
\end{tabular}

*statistically significant change

\section{Discussion}

This study demonstrated that eight 2 min karate fights increased AT stiffness in the dominant leg. The present study was the first to investigate changes in AT stiffness as the effect of karate fights.

Our research indicates that AT stiffness increased after a specific karate load. It can be the effect of multiple tensile strain cycles that the AT undergoes during a fight (footwork, jumps, changing distance and kicking). Our observation is in line with the latest research focused on the acute effect of AT loading. Joseph et al. (2015) showed that repeated cyclical loading of downhill backwards walking affected the behaviour of the muscle-tendon unit, most likely by altering muscle compliance, and these changes resulted in a tendon stiffness increase. Another study of Morrissey et al. (2011) demonstrated that 6 weeks of eccentric training decreased tendon stiffness in individuals with healthy ATs, however, a similar change was not observed in the concentric group, which could be the reason why concentric nature of the tendon load during fights increased AT stiffness in our experiment. This is only a speculation and it needs further research (Morrissey et al., 2011).

In the literature we can find various results regarding the influence of training on AT characteristics. Kurihara et al. (2012) showed that there was no significant difference of AT stiffness between various groups of different sports activities. They suggested that the mechanical characteristics of the AT were unchanged with the load characteristics of each sport. Other authors showed significant hypertrophy due to training (Ker et al., 2011).

It should be underlined that numerous researchers have studied the elastic properties of $\mathrm{AT}$, but they used different research methods, thus, it is difficult to directly compare their results with ours. Bobbert et al. (1990) used a rat tendon model and showed that tendon structure was highly related to tendon compliance in that long thin tendons were more compliant than short thick tendons. Diggin et al. (2016) assessed "lower-limb muscle-tendon stiffness" using a model based on two trials of single legged hopping at 1.5, 2.2 and $3.0 \mathrm{~Hz}$ at the same time of day across three days. Other researchers used shear wave elastography and showed that muscle 
elasticity was associated with passive joint stiffness in the joint position where the muscle was sufficiently lengthened (Chino et al., 2011).

Finally, we should recognize the limitations of this study. First, a greater sample size is needed to permit a more generalized interpretation of these results. Second, the fact that no control group took part in the present study might be seen as a drawback. However, in the future studies we will focus on the AT of untrained subjects and athletes from other disciplines. Moreover, further investigations in other tendons and muscles are needed to enable a generalization of the current findings.
Additional research on AT using myotonometry is clearly needed. Precise evaluation of AT stiffness is very important from a practical point of view. During stretch shortening cycle movements, such as vertical jumping, the ability to produce force rapidly is vital to athletic performance. Understanding the relationship between tendon structure and these abilities can increase the efficacy of talent identification and provide practitioners with the ability to prescribe specific strength, conditioning, physiotherapy and injury prevention programs that focus on structural adaptations.

\section{References}

Bobbert MF, Ettema GC, Huijing PA. The force-length relationship of a muscle-tendon complex: experimental results and model calculations. Eur J Appl Physiol Occup Physiol, 1990; 61: 323329

Chuang LL, Wu CY, Lin KC, Lur SY. Quantitative mechanical properties of the relaxed biceps and triceps brachii muscles in patients with subacute stroke: a reliability study of the myoton-3 myometer. Stroke Res Treat, 2012; 617-694

Chino K, Takahashi H. Measurement of gastrocnemius muscle elasticity by shear wave elastography: association with passive ankle joint stiffness and sex differences. Eur J Appl Physiol, 2016 Feb 13. [Epub ahead of print]

Diggin D, Anderson R, Harrison AJ. An Examination of the True Reliability of Lower Limb Stiffness Measures During Overground Hopping. J Appl Biomech, 2016 Jan 7. [Epub ahead of print]

Ditroilo M, Hunter AM, Haslam S, De Vito G. The effectiveness of two novel techniques in establishing the mechanical and contractile responses of biceps femoris. Physiol Meas, 2011; 32: 1315-26

Fouré A, Nordez A, Cornu C. Effects of plyometric training on passive stiffness of gastrocnemii muscles and Achilles tendon. Eur J Appl Physiol, 2012; 112(8): 2849-57

Fouré A, Nordez A, Cornu C. Effects of eccentric training on mechanical properties of the plantar flexor muscle-tendon complex. J Appl Physiol, 2013; 1; 114(5): 523-37

Joseph CW, Bradshaw EJ, Furness TP, Kemp J, Clark RA. Early changes in Achilles tendon behaviour in vivo following downhill backwards walking. J Sports Sci, 2015; 29: 1-7. [Epub ahead of print]

Ker RF, Wang XT, Pike AV. Fatigue quality of mammalian tendons. J. Exp. Bio, 2000; 203: 1317-1327

Komi PV, Fukashiro S, Järvinen M. Biomechanical loading of Achilles tendon during normal locomotion. Clin Sports Med, 1992; 11: 521 - 531

Kubo K, Kanehisa H, Kawakami Y, Fukunaga T. Elasticity of tendon structures of the lower limbs in sprinters. Acta Physiologica Scandinavica, 2000; 168 (2): 327-335

Kujala UM, Taimela S, Anti-Poikka I, Orava S, Tuominen R, Myllynen P. Acute injuries in soccer ice 
hockey, volleyball, basketball, judo, and karate: analysis of national registry data. BMJ, 1995; 311(7018): 1465-8

Kurihara T, Sasaki R, Isaka T. Mechanical properties of achilles tendon in relation to various sport activities of collegiate athletes. 30 th Annual Conference of Biomechanics in Sports. Melbourne, 183; 2012

Marusiak J, Jaskólska A, Budrewicz S, Koszewicz M, Jaskólski A. Increased muscle belly and tendon stiffness in patients with Parkinson's disease, as measured by myotonometry. Mov Disord, 2011; 26(11): 2119-22

Morrissey D, Roskilly A, Twaycross-Lewis R, Screen H, Woledge R, Bader D. The effect of eccentric and concentric calf muscle training on Achilles tendon stiffness. Clinical Rehabilitation 2011: 25: 238-247

Park DY, Chou L. Stretching for prevention of Achilles tendon injuries: a review of the literature. Foot Ankle Int, 2006; 27: 1086 - 95

Rettig AC, Liotta FJ, Klootwyk TE, Porter DA, Mieling P. Potential risk of rerupture in primary achilles tendon repair in athletes younger than 30 years of age. Am J Sports Med, 2005; 33(1): $119-23$

Sterkowicz S, Sterkowicz-Przybycień K. Injuries in karate: A review. OA Sports Medicine, 2013; 1(2): 14

Wang HK, Lin KH, Su SC, Shih TT, Huang YC. Effects of tendon viscoelasticity in Achilles tendinosis on explosive performance and clinical severity in athletes. Scand J Med Sci Sports, 2012; 22(6): 147-55

\section{Corresponding author:}

\section{Adam Kawczyński, PhD}

University School of Physical Education al. I.J. Paderewskiego 35, Wrocław, Poland

Phone: +48 606230553

E-mail: kawczynski.a@gmail.com 